\title{
Elevated preoperative neutrophil-to-lymphocyte ratio is associated with poor prognosis in gastrointestinal stromal tumor patients
}

\author{
Chang Jiang ${ }^{1-3, *}$ \\ Wan-Ming $\mathrm{Hu}^{2-4, *}$ \\ Fang-Xin $\mathrm{LiaO}^{\mathrm{I}-3}$ \\ Qiong Yang ${ }^{1-3}$ \\ Ping Chen ${ }^{1-3}$ \\ Yu-Ming Rong ${ }^{1-3}$ \\ Gui-Fang Guo $0^{1-3}$ \\ Chen-XiYin 2,3,5 \\ Bei Zhangl-3 \\ Wen-Zhuo $\mathrm{He}^{1-3}$ \\ Liang-Ping $\mathrm{Xia}^{1-3}$
}

'VIP Department, Sun Yat-sen University Cancer Center, ${ }^{2}$ State

Key Laboratory of Oncology

in South China, ${ }^{3}$ Collaborative

Innovation Center for Cancer

Medicine, ${ }^{4}$ Department of Pathology,

${ }^{5}$ Intensive Care Unit, Sun Yat-sen

University Cancer Center, Guangzhou,

Guangdong, People's Republic of

China

*These authors contributed equally to this work
This article was published in the following Dove Press journal:

OncoTargets and Therapy

23 February 2016

Number of times this article has been viewed

Purpose: To investigate the prognostic relevance of preoperative peripheral neutrophilto-lymphocyte ratio (NLR) in gastrointestinal stromal tumor (GIST) patients.

Materials and methods: We enrolled 129 consecutive GIST patients who underwent initial curative surgical resection with or without adjuvant/palliative imatinib treatment in our study. Blood NLR was calculated as neutrophil count (number of neutrophils $\times 10^{9} / \mathrm{L}$ ) divided by lymphocyte count (number of lymphocytes $\times 10^{9} / \mathrm{L}$ ). Survival curves were constructed by using the Kaplan-Meier method. Univariate and multivariate Cox proportional hazard regression models were performed to identify associations with outcome variable. All tests were two-sided, and $P<0.05$ was considered statistically significant.

Results: The optimal cut-off value of NLR was 2.07 in the receiver operating characteristic curve analysis. The median overall survival (OS) of high NLR group was 113.0 months, whereas that of the low NLR group had not reached the median OS both in the general $(P<0.001)$ and subgroup analyses. The elevated NLR suggested shorter OS in the high malignant potential groups $(P=0.01)$ and the combined low and moderate groups $(P=0.02)$. Increased NLR indicated poor OS in patients regardless of whether if received imatinib treatment or not $(P=0.005$, and $P=0.032$, respectively). High NLR indicated poor OS of patients in stage I and II disease $(P=0.005)$ and a clear tendency that increased level of NLR is inimical to OS.

Conclusion: Elevated NLR was detected as an independent adverse prognostic factor. Elevated preoperative NLR predicts poor clinical outcome in GIST patients and may serve as a costeffective and broadly available independent prognostic biomarker.

Keywords: neutrophil-to-lymphocyte ratio, overall survival, biomarker, GIST

\section{Introduction}

Gastrointestinal stromal tumor (GIST) is the most common subtype of gastrointestinal sarcoma, ${ }^{1}$ arising from the precursor cells of the interstitial cells of Cajal in the gastrointestinal tract, ${ }^{2}$ with a estimated annual incidence of 1.5 cases per 100,000 persons. ${ }^{3}$ The incidence of GISTs occurring in the stomach is $50 \%$, and that occurring in the jejunum and ileum for is $25 \%$. However, GISTs can also occur in the rest gastrointestinal tract as well as within the peritoneum. ${ }^{4}$ Approximately $85 \%$ of GISTs contain a function mutation in the KIT, whereas 3\%-5\% can have a mutation in platelet-derived growth factor receptor $\alpha$ (PDGFR $\alpha){ }^{5}$ The standard treatment for localized, primary gastrointestinal stromal tumor is complete surgical excision, without the dissection of clinically negative lymph nodes. ${ }^{6}$ However, up to $50 \%$ patients treated with surgery alone will develop tumor recurrence within 5 years and eventually die from the disease. ${ }^{5}$ The progress of small molecule tyrosine kinase inhibitors such as imatinib that

\footnotetext{
Correspondence: Liang-Ping Xia; Wen-Zhuo He

VIP Department, Sun Yat-sen University Cancer Center, 65I Dongfeng East Road, Guangzhou 510060, Guangdong, People’s Republic of China

Tel +862087343107

Fax +86 2087343392

Email xialiangping@163.com; happyhewenzhuo@I63.com
}

(c) (7) (3) 2016 jiang et al. This work is published and licensed by Dove Medical Press Limited. The full terms of this license are available at https://www.dovepress.com/terms.php cc) hereby accept the Terms. Non-commercial uses of the work are permitted without any further permission from Dove Medical Press Limited, provided the work is properly attributed. For permission for commercial use of this work, please see paragraphs 4.2 and 5 of our Terms (https://www.dovepress.com/terms.php). 
selectively inhibits KIT, PDGFR $\alpha, \mathrm{ABL}$, and BCR-ABL, has substantially improved the prognosis of GIST. ${ }^{7}$ The widely used risk classification incorporates the primary tumor site, mitotic count, and tumor size, which are the three main prognostic factors in localized GIST. ${ }^{8}$ In addition, tumor rupture (either at surgery or spontaneously) is an independent risk factor that negatively impacts prognosis. ${ }^{9}$

The tumor microenvironment, particularly the inflammatory and immune response, plays an important role in cancer development, ${ }^{10}$ including GISTs. ${ }^{11}$ The peripheral neutrophilto-lymphocyte ratio (NLR) is an accessible, reproducible marker of systemic inflammation. Recently, elevated NLR has been associated with an adverse overall survival (OS) in various solid tumors, such as colorectal cancer, ${ }^{12}$ gastric cancer, ${ }^{13}$ renal cell carcinoma, ${ }^{14}$ non-small-cell lung cancer, ${ }^{15}$ and ovarian cancer. ${ }^{16}$ Perez et al ${ }^{17}$ revealed that high-NLR was associated with poor recurrence-free survival (RFS) in resectable localized GIST, with the exclusion of patients who received adjuvant imatinib treatment. However, it is unclear whether NLR, the readily available biomarker, correlates with OS in GIST.

In our study, we retrospectively analyzed the untreated, primary GIST patients who received curative surgery (surgical resection with curative intent). We used preoperative NLR as-prognostic factor in these patients. The receiver operating characteristic (ROC) curve was used to determine the adequate cut-off value with the best sensitivity and specificity. Preoperative NLR was used to identify patients with better or worse prognosis. Its role as marker for OS was assessed and a potential correlation with established risk criteria was analyzed. ${ }^{8,18}$

\section{Materials and methods Patients}

Patients were eligible to participate in the study if they met the following criteria: 1) initially diagnosed with primary resectable GIST at the Sun Yat-sen University Cancer Center (SYSUCC) between 2000 and 2010; 2) had Eastern Cooperative Oncology Group (ECOG) status $\leq 2$ 3) had available records of complete blood count before any treatment; 4) underwent initial curative surgical resection with or without adjuvant/palliative imatinib treatment; and 5) had available follow-up data. Patients with other types of synchronous tumors or incomplete set of blood values were excluded. Preoperative extent of disease was assessed by using abdominal/pelvic computed tomography or magnetic resonance imaging. The procedures of the study were approved by the Sun Yat-Sen Cancer Center Committee. Written consent was obtained from all participants.

\section{Peripheral blood counts}

Peripheral blood samples were obtained from each patient between 8 and 10 o'clock in the morning, within 10 days before surgery. The collected samples were translocated to sterile centrifuge tubes, which were then carefully delivered to the department of clinical laboratory. The complete blood counts were conducted by using the XE-5000TM Automated Hematology System (Sysmex Corporation, Shanghai, People's Republic of China). None of the patients had clinical signs of infection at the time of blood sampling. Blood NLR was calculated as neutrophil count (number of neutrophils $\times 10^{9} / \mathrm{L}$ ) divided by lymphocyte count (number of lymphocytes $\times 10^{9} / \mathrm{L}$ ).

\section{Pathological diagnose}

Tumor histology was established by the Department of Pathology of SYSUCC. For diagnosis of GIST, formalinfixed, paraffin-embedded tumor samples were immunohistochemically stained by using standard protocols. ${ }^{19}$ Tumor size, site, and mitotic rate (number of mitoses per 50 highpower fields) were recorded.

\section{Statistical analysis}

Statistical analyses were performed by using the SPSS software (version 18.0; SPSS Inc., Chicago, IL, USA). Because the NLR cut-off value for GIST prognosis was inconsistent, we tried to find the best one. The distributions of the baseline characteristics of the patients were assessed by the $\chi^{2}$ test. ${ }^{20}$ OS was defined as the time from diagnosis to the date of death or the last date of follow-up. The survival curves were calculated by using the Kaplan-Meier method. Univariate and multivariate Cox proportional hazard regression models were performed to identify associations with outcome variables. ${ }^{21}$ All tests were two-sided, and $P<0.05$ was considered statistically significant.

\section{Results ROC analysis}

We used the continuous variable NLR as the test variable, and $\mathrm{OS}$ as the state variable. The area under the ROC curve of NLR was 0.736 (95\% confidence interval [CI]: $0.646-0.847 ; P<0.001$; Figure 1). For all of the GIST patients, NLR $=2.07$ had the highest sensitivity (78.4\%) and specificity $(57.6 \%)$.

\section{Patient characteristics}

We enrolled 129 consecutive patients in this retrospective study. The median age of the entire population at diagnosis 


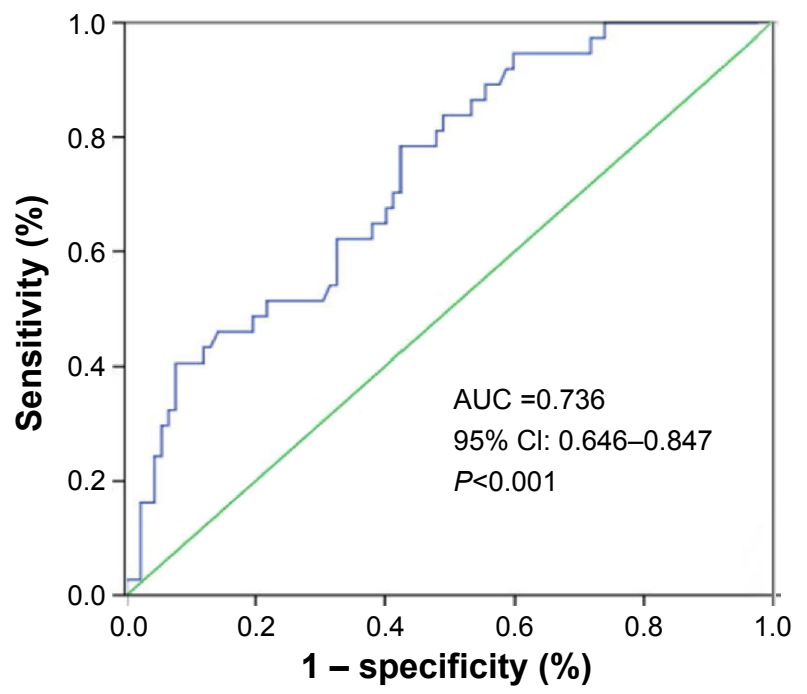

Figure I Receiver operating characteristic curves for neutrophil-to-lymphocyte ratio (NLR) to predict overall survival (OS).

Notes: In a study of I 29 GIST patients, blood NLR was calculated as neutrophil count $\left(\times 10^{9} / \mathrm{L}\right)$ divided by lymphocyte count $\left(\times 10^{9} / \mathrm{L}\right)$ by using the results of complete blood counts before surgery. A receiver-operating characteristic graph was constructed to estimate the optimal cut-off value. The area under the receiver-operating characteristic curve of NLR was 0.736 . The NLR of 2.07 had the highest sensitivity and specificity. The green line represents the reference line. The blue line represents NLR.

Abbreviations: AUC, area under the receiver-operating characteristic curve; $\mathrm{Cl}$, confidence interval; GIST, gastrointestinal stromal tumor.

was 55 years (range: $20-86$ years) and $60.5 \%$ (78/129) of the patients were male. The most common location was the stomach $(50.4 \% ; 65 / 129)$, followed by the jejunum and ileum $(24 \% ; 31 / 129)$, colon and rectum $(7.8 \% ; 10 / 129)$, duodenum (4.7\%; 6/129), esophagus $(3.9 \% ; 5 / 129)$, peritoneum $(3.9 \%$; $5 / 129)$ and other sites $(5.3 \% ; 7 / 129)$. The median mitotic rate was 5.5 mitoses/50 HPF (interquartile range: $3-15.75$ ) and the median tumor size was $7 \mathrm{~cm}$ (interquartile range: 4-12). Twelve patients had metastatic lesions in liver, enterocelia, pelvic cavity, or peritoneum that were detected during operation. Table 1 shows the baseline parameters stratified according to NLR in all of the patients before treatment.

\section{Survival analyses}

The high NLR group had a median OS of 113.0 (113.0 \pm 21.2 , 95\% CI: 74.5-154.5) months, whereas the low NLR group had not reached the median OS both in the general $(P<0.001$; Figure 2) and subgroup analyses. The patients were classified as having low $(n=34)$, moderate $(n=12)$, or high $(n=83)$ malignant potential according to established criteria. ${ }^{8}{ }^{818} \mathrm{In}$ the high malignant potential group, the median OS of the patients with high NLR was 71.8 (71.8 \pm 29.3 ; 95\% CI: 14.4-129.1) months, and the elevated NLR suggested shorter OS ( $P=0.01$; Figure 3). Despite that the numbers of patients in the low and moderate groups were not adequate for Kaplan-Meier survival analysis, respectively, and that the median OS was
Table I Patient characteristics

\begin{tabular}{|c|c|c|c|}
\hline Characteristics & $\begin{array}{l}\text { Low NLR (\%), } \\
n=61\end{array}$ & $\begin{array}{l}\text { High NLR (\%), } \\
n=68\end{array}$ & $P$-value \\
\hline \multicolumn{4}{|l|}{ Sex } \\
\hline Male & $32(52.5)$ & $46(67.6)$ & \\
\hline Female & $29(47.5)$ & $22(32.4)$ & 0.08 \\
\hline \multicolumn{4}{|l|}{ Age (years) } \\
\hline Median (range) & $55(20-89)$ & $55(21-80)$ & \\
\hline$<60$ & $43(70.5)$ & $44(64.7)$ & \\
\hline$>60$ & $18(29.5)$ & $24(35.3)$ & 0.48 \\
\hline \multicolumn{4}{|l|}{ ECOG PS } \\
\hline 0 & $28(45.9)$ & $21(30.9)$ & \\
\hline 1 & $32(52.5)$ & $45(66.2)$ & \\
\hline 2 & I (I.6) & $2(2.9)$ & 0.21 \\
\hline \multicolumn{4}{|l|}{ Tumor size $(\mathrm{cm})$} \\
\hline$<5$ & $27(44.3)$ & $21(30.9)$ & \\
\hline$>5$ & $34(55.7)$ & $47(69.1)$ & 0.15 \\
\hline \multicolumn{4}{|c|}{ Mitotic rate (per 50 HPFs) } \\
\hline$<5$ & $38(62.3)$ & $31(45.6)$ & \\
\hline$>5$ & $23(37.7)$ & $37(54.4)$ & 0.08 \\
\hline \multicolumn{4}{|l|}{ Location } \\
\hline Stomach & $35(57.4)$ & $30(44.1)$ & \\
\hline Jejunum and ileum & $9(14.8)$ & $22(32.4)$ & \\
\hline Colon and rectum & 7 (II.5) & $3(4.4)$ & \\
\hline Other ${ }^{a}$ & $10(16.3)$ & $13(19.1)$ & 0.08 \\
\hline \multicolumn{4}{|l|}{ Stage } \\
\hline $\mathrm{I}^{\mathrm{b}}$ & $27(44.3)$ & $16(23.5)$ & \\
\hline ॥ & $14(23)$ & II (16.2) & \\
\hline IIIc & I 7 (27.8) & $32(47.1)$ & \\
\hline $\mathrm{IV}^{\mathrm{d}}$ & $3(4.9)$ & $9(\mid 3.2)$ & 0.02 \\
\hline \multicolumn{4}{|l|}{ Malignant potential } \\
\hline Low & $20(32.8)$ & $14(20.6)$ & \\
\hline Moderate & $8(13.1)$ & $4(5.9)$ & \\
\hline High & $33(54.1)$ & $50(73.5)$ & 0.06 \\
\hline Imatinib treatment $\mathrm{t}^{\mathrm{e}}$ & $27(44.3)$ & $30(44.1)$ & 0.97 \\
\hline
\end{tabular}

Notes: ancluding the duodenum (4 in the low NLR group, 2 in the high NLR group), esophagus ( 5 in the high NLR group), peritoneum (2 in the low NLR group, 3 in the high NLR group), omentum (I in the low NLR group, I in the high NLR group), pancreas (I in the high NLR group), unknown (2 in the low NLR group and I in the high NLR group). Including stage IA and IB diseases. Including stage IIIA and IIIB diseases. 'Metastatic lesions located in liver, enterocelia, pelvic cavity and peritoneum. elmatinib treatment containing adjuvant and palliative therapy.

Abbreviations: ECOG PS, Eastern Cooperative Oncology Group performance status; HPFs, high-power fields; NLR, neutrophil-to-lymphocyte ratio.

not reached in these patients, the increased NLR still implied unfavorable OS in the combined low and moderate malignant potential groups ( $P=0.02$; Figure 4$)$. Moreover, high NLR indicated poor OS regardless of whether or not the patients received imatinib treatment $(113 \pm 33.3$ months in high NLR group, $P=0.005$; Figure 5 , and $103 \pm 22.3$ months in high NLR group, $P=0.032$; Figure 6 ). Considering that more patients in the low NLR group were diagnosed with stage I or II disease and the plurality of patients with elevated NLR distributed in stage III or IV, we analyzed the relationship between NLR and OS in stages I and II, III, and IV, respectively. High NLR indicated poor OS in the patients with stage I and II 


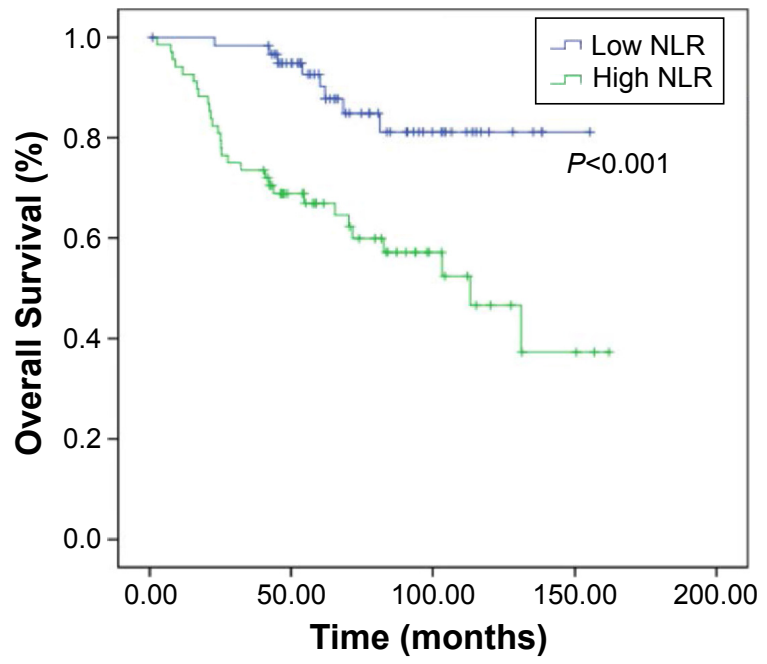

Figure 2 Overall survival (OS) of GIST patients classified by NLR.

Notes: Kaplan-Meier estimate of the censoring time distribution in 129 GIST patients. High NLR was significantly associated with shorter overall survival. The $P$-value was calculated by using the log-rank test.

Abbreviations: GIST, gastrointestinal stromal tumor; NLR, neutrophil-to-lymphocyte ratio.

diseases $(P=0.005$; Figure 7$)$, although all of the patients with stage I and II diseases had not reached the median OS. We could also detect a clear tendency that increased level of NLR is inimical to OS $(82.7 \pm 26.3$ months in high NLR group, $P=0.058$; Figure 8 ).

In univariate analysis, NLR (hazard ratio [HR]: 4.08; 95\% CI: 1.65-10.06; $P=0.001)$, NLR, stage, tumor size, mitotic rate, location, and malignant potential were associated with

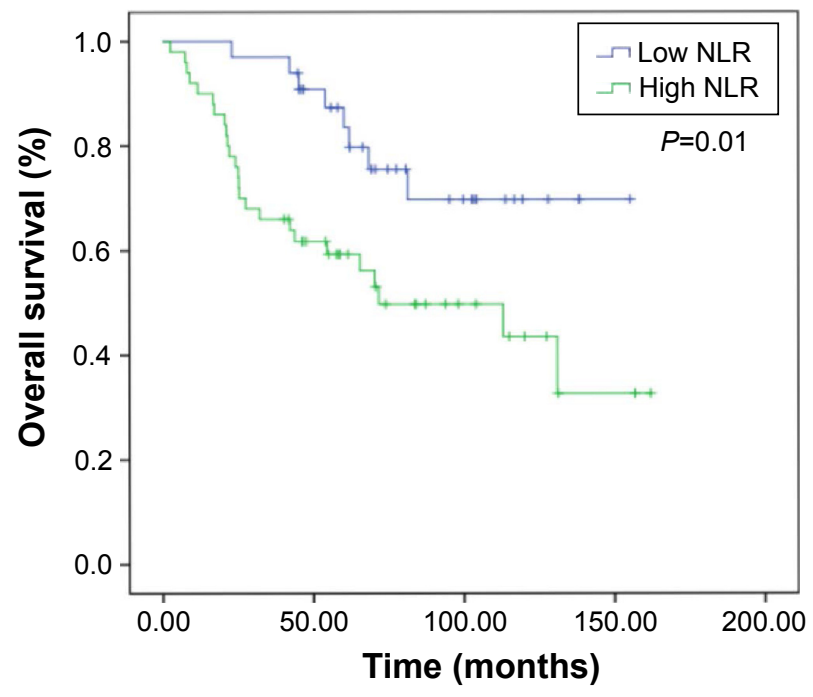

Figure 3 OS of high malignant potential GIST patients classified by NLR. Note: In the study of 83 GIST patients with high malignant potential, elevated NLR suggested shorter OS, and the patients with low NLR group did not reach the median OS.

Abbreviations: GIST, gastrointestinal stromal tumor; NLR, neutrophil-to-lymphocyte ratio; OS, overall survival.

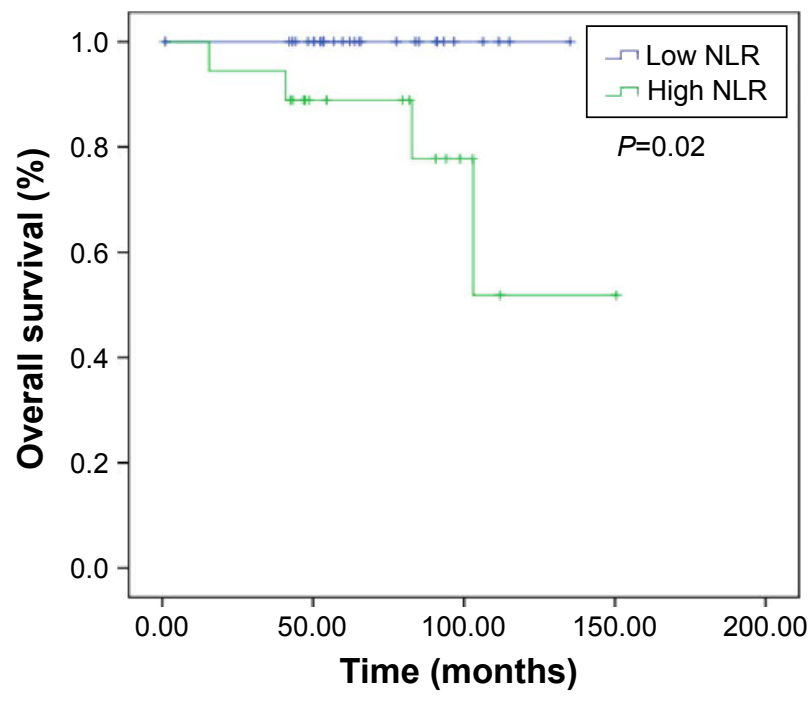

Figure 4 OS of GIST patients in combined groups classified by NLR.

Note: In the GIST patients with combined low and moderate malignant potential, increased NLR implied unfavorable OS.

Abbreviations: GIST, gastrointestinal stromal tumor; NLR, neutrophil-to-lymphocyte ratio; OS, overall survival.

OS (Table 2). Moreover, multivariate analysis identified only NLR (HR: 3.13, 95\% CI: $1.72-10.08, P=0.009$ ) and stage as independent predictive factors. Elevated NLR was detected as an independent adverse prognostic factor.

\section{Discussion}

Various studies have suggested that elevated NLR is associated with poor survival. Templeton et al discovered a consistent



Figure 5 OS of patients without imatinib treatment.

Note: In the subgroup analysis of 72 patients who did not receive imatinib treatment, high NLR correlated with shorter OS.

Abbreviations: NLR, neutrophil-to-lymphocyte ratio; OS, overall survival. 


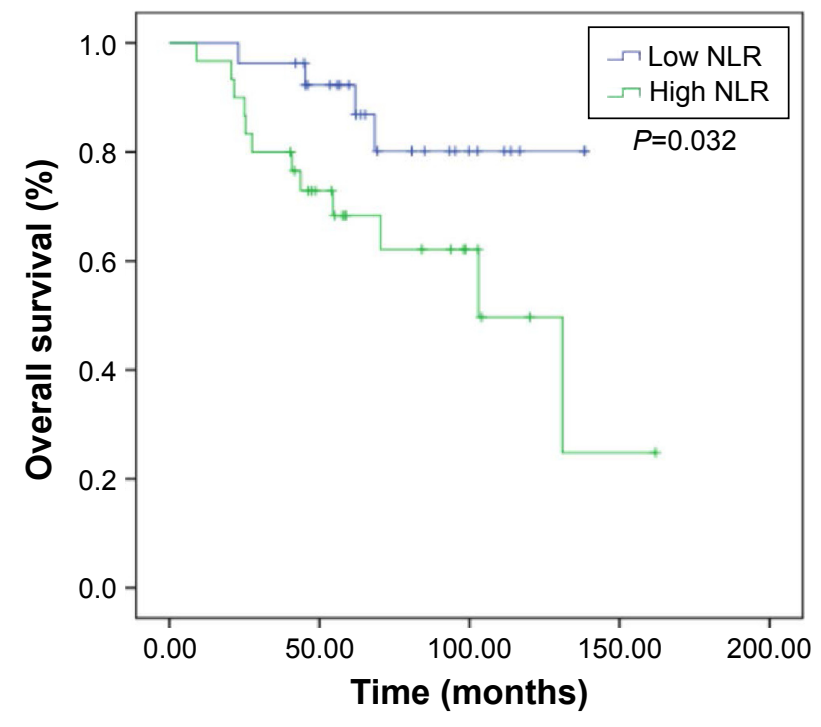

Figure 6 OS of patients with imatinib treatment.

Note: In the subgroup analysis of 57 patients who received imatinib treatment, high NLR was associated with shorter OS.

Abbreviations: NLR, neutrophil-to-lymphocyte ratio; OS, overall survival.

effect of elevated NLR on survival ( $H R=1.81)$ among various solid tumors and across disease sites and stages. ${ }^{22}$ In this study, we observed the prognostic impact of NLR on OS. It is interesting that diverse cut-off NLRs for different cancers were reported. For GIST, Perez et al chose the sample median NLR as a cut-off. ${ }^{17}$ As nuances may exit among the reference values of neutrophil and lymphocyte in multiple clinical laboratories, we used ROC to determine the optimal cut-off value.

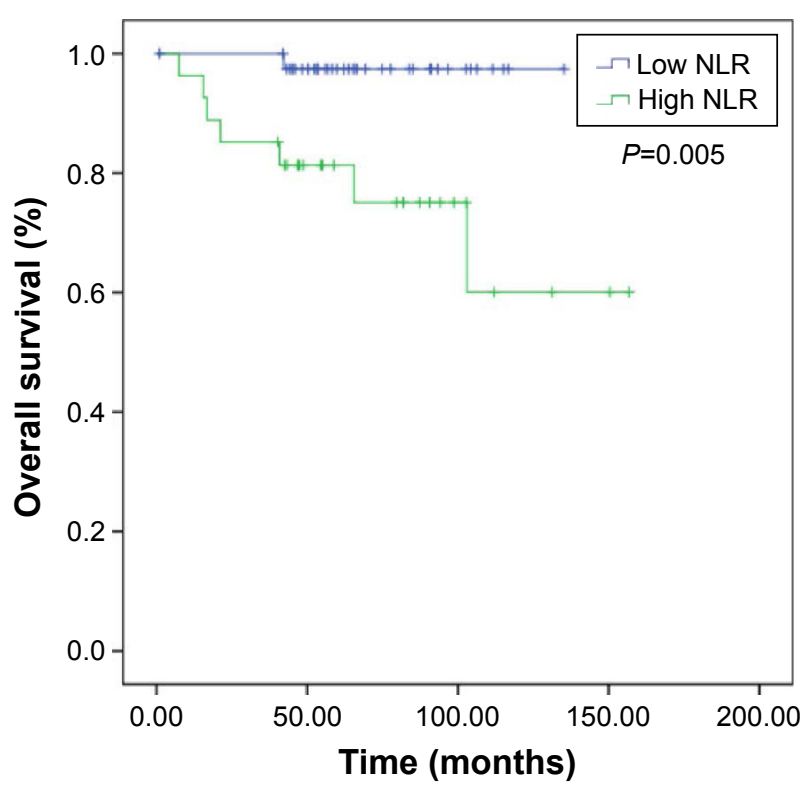

Figure 7 OS of patients in stage I and II.

Notes: In view of the unbalanced distribution of stages in the two groups, a subgroup analysis within the stages was performed. High NLR was associated with poorer OS in the patients with stage I and II diseases.

Abbreviations: NLR, neutrophil-to-lymphocyte ratio; OS, overall survival.

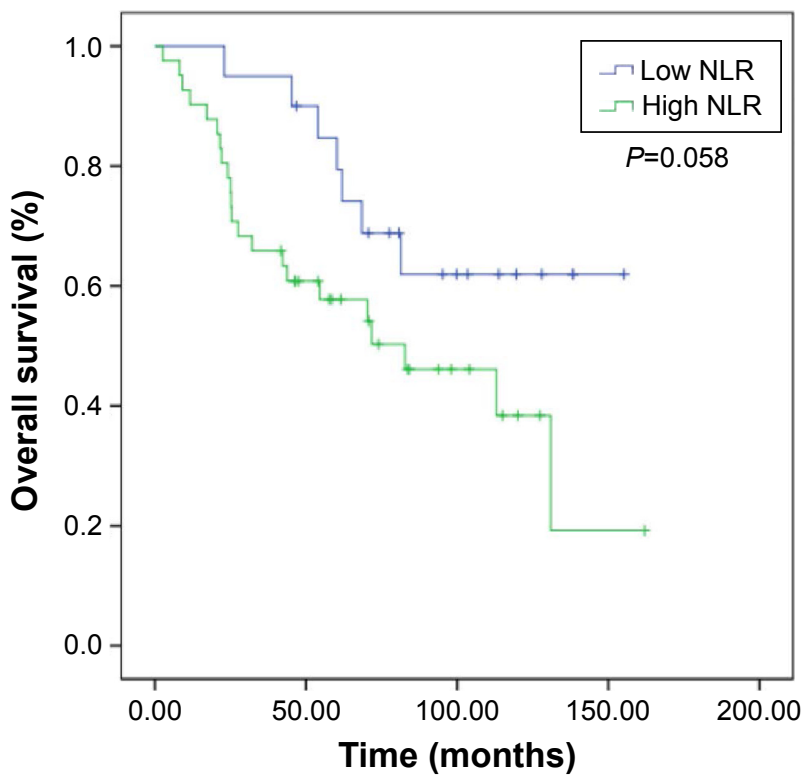

Figure 8 OS of patients in stage III and IV.

Note: In the patients with stage III and IV diseases, a clear tendency showed that an increased NLR was inimical to OS.

Abbreviations: NLR, neutrophil-to-lymphocyte ratio; OS, overall survival.

In the study of GISTs conducted by Perez et al, ${ }^{17}$ which revealed the negative correlation between NLR and RFS, the authors had to exclude patients who had received adjuvant imatinib therapy to reduce the bias in RFS. This means the patients enrolled in their study mostly had early-stage disease and low and moderate risk of recurrence. However, our work included patients with stage I to II disease with various malignant potential. As an unfavorable prognostic factor, elevated NLR was found to be a valuable predictive parameter for tumor staging in colorectal cancer patients. ${ }^{23}$ We discovered that higher NLR was associated with higher prevalence of stage III and IV diseases and with high malignant potential. Howbeit, our data are consistent with the fact that increased NLR was associated with tumor features

Table 2 Cox regression analysis for OS

\begin{tabular}{|c|c|c|c|c|c|c|}
\hline \multirow[t]{2}{*}{ Variable } & \multicolumn{3}{|c|}{ Univariate } & \multicolumn{3}{|c|}{ Multivariate } \\
\hline & $P$-value & HR & $95 \% \mathrm{Cl}$ & $P$-value & HR & $95 \% \mathrm{Cl}$ \\
\hline NLR & 0.001 & 4.08 & $1.65-10.06$ & 0.006 & 3.22 & I.39-7.43 \\
\hline Stage & $<0.001$ & 2.44 & $1.64-3.64$ & 0.018 & 2.34 & $1.16-4.73$ \\
\hline Tumor size & 0.020 & 2.14 & $1.12-4.09$ & 0.310 & 1.45 & $0.71-2.99$ \\
\hline Mitotic rate & 0.003 & 2.91 & $1.44-5.90$ & 0.104 & 1.90 & $0.88-4.13$ \\
\hline Location $^{\mathrm{a}}$ & 0.011 & 2.51 & $1.23-5.1 \mathrm{I}$ & 0.183 & 1.66 & $0.79-3.52$ \\
\hline Imatinib & 0.181 & 0.66 & $0.36-1.21$ & 0.083 & 0.54 & $0.27-1.08$ \\
\hline $\begin{array}{l}\text { Malignant } \\
\text { potential }\end{array}$ & 0.005 & 2.38 & I.30-4.34 & 0.863 & 0.91 & $0.33-2.54$ \\
\hline
\end{tabular}

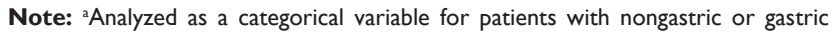
stromal tumor.

Abbreviations: $\mathrm{Cl}$, confidence interval; $\mathrm{HR}$, hazard ratio; NLR, neutrophil-tolymphocyte ratio; OS, overall survival. 
indicating high malignant potential and possibly with tumor staging in GIST. ${ }^{17}$

The mechanisms underlying the association between high NLR and poor outcome of cancer patients remain obscure. One potential mechanism may be an association between high NLR with tumor promoting inflammation and immune response, including increased neutrophil and decreased lymphocyte, which are regarded as a hallmark of cancer. ${ }^{24}$ Increased number of tumor-infiltrating lymphocytes had been associated with better prognosis. ${ }^{25,26}$ In addition, several tumor-related antigens had been identified as prognostic predictors. ${ }^{27}$ Balachandran et al found that GISTs induce an intratumoral T-cell immune response suppression by secreting an immunosuppressive enzyme (indoleamine 2,3-dioxygenase). ${ }^{11}$

Meanwhile, as an inflammatory response, accumulated neutrophils have been associated with poor clinical outcome. ${ }^{15}$ First, increased neutrophils count inhibits the immune system via restraining the cytolytic activity of immune cells such as lymphocytes, activated T-cells, and natural killer cells. ${ }^{28,29}$ Second, neutrophils had been reported to influence the tumor environment by means of secreting tumor growth promoting factors as vascular endothelial growth factor, ${ }^{30}$ hepatocyte growth factor, ${ }^{31}$ interleukin (IL)- $6,{ }^{32}$ and IL- $8^{33}$ and thus likely contribute to stimulating tumor angiogenesis and progression. To some extent, the NLR indicates an interaction between pro-tumor inflammatory response and anti-tumor immune response. ${ }^{34}$ With elevated NLR, The inflammatory status seems to be dominant and generates poor outcome.

NLR had been incorporated in a simple score for men with metastatic castration-resistant prostate cancer because of providing independent prognostic information. ${ }^{35}$ As an available and affordable index, NLR might become a biomarker to forecast the therapeutic effect and prognosis in GIST.

This study has several limitations. According to the National Comprehensive Cancer Network guideline, patients who undergo completely resection without significant risk of recurrence or preoperative imatinib are not required to receive adjuvant imatinib treatment. Adjuvant imatinib therapy prolongs RFS and may improve OS. ${ }^{7}$ However, in clinical practice, not all patients eligible for imatinib treatment received imatinib or were treated with a standard duration. This might have resulted in imatinib as an insignificant factor in both univariate and multivariate analyses. The nature of this study being retrospective and non-randomized, and its small patient samples might have led to the failure of the traditionally considered prognostic factors such as tumor size, site, and mitotic rate. ${ }^{8,18}$

\section{Conclusion}

The NLR is an independent factor associated with adverse survival in GIST and may serve as a cost-effective prognostic biomarker. Large scale prospective studies are needed to verify our findings. The utility of NLR for therapeutic decision-making should be evaluated.

\section{Acknowledgments}

This work was supported by the the Natural Science Foundation of Guangdong, China (2015A030313010), Science and Technology Program of Guangzhou, China (1563000305), and National Natural Science Foundation of China (81272641and 81572409).

\section{Disclosure}

The authors report no conflicts of interest in this work.

\section{References}

1. Miettinen M, Lasota J. Gastrointestinal stromal tumors - definition, clinical, histological, immunohistochemical, and molecular genetic features and differential diagnosis. Virchows Arch. 2001;438(1):1-12.

2. Corless CL, Fletcher JA, Heinrich MC. Biology of gastrointestinal stromal tumors. J Clin Oncol. 2004;22(18):3813-3825.

3. Gatta G, van der Zwan JM, Casali PG, et al. Rare cancers are not so rare: the rare cancer burden in Europe. Eur J Cancer. 2011;47(17): 2493-2511.

4. Choi H, Charnsangavej C, Faria SC, et al. Correlation of computed tomography and positron emission tomography in patients with metastatic gastrointestinal stromal tumor treated at a single institution with imatinib mesylate: proposal of new computed tomography response criteria. J Clin Oncol. 2007;25(13):1753-1759.

5. Dematteo RP, Ballman KV, Antonescu CR, et al. Adjuvant imatinib mesylate after resection of localised, primary gastrointestinal stromal tumour: a randomised, double-blind, placebo-controlled trial. Lancet. 2009;373(9669):1097-1104.

6. ESMO/European Society for Medical Oncology. Gastrointestinal stromal tumors: ESMO Clinical Practice Guidelines for diagnosis, treatment and follow-up. Ann Oncol. 2012;23 Suppl 7:vii49-vii55.

7. Joensuu H, DeMatteo RP. The management of gastrointestinal stromal tumors: a model for targeted and multidisciplinary therapy of malignancy. Аnпu Rev Med. 2012;63:247-258.

8. Miettinen M, Lasota J. Gastrointestinal stromal tumors: review on morphology, molecular pathology, prognosis, and differential diagnosis. Arch Pathol Lab Med. 2006;130(10):1466-1478.

9. Rutkowski P, Nowecki ZI, Michej W, et al. Risk criteria and prognostic factors for predicting recurrences after resection of primary gastrointestinal stromal tumor. Ann Surg Oncol. 2007;14(7):2018-2027.

10. Grivennikov SI, Greten FR, Karin M. Immunity, inflammation, and cancer. Cell. 2010;140(6):883-899.

11. Balachandran VP, Cavnar MJ, Zeng S, et al. Imatinib potentiates antitumor $\mathrm{T}$ cell responses in gastrointestinal stromal tumor through the inhibition of Ido. Nat Med. 2011;17(9):1094-1100.

12. $\mathrm{He} \mathrm{W}$, Yin $\mathrm{C}, \mathrm{Guo} \mathrm{G}$, et al. Initial neutrophil lymphocyte ratio is superior to platelet lymphocyte ratio as an adverse prognostic and predictive factor in metastatic colorectal cancer. Med Oncol. 2013;30(1):439.

13. Dutta $\mathrm{S}$, et al. Comparison of the prognostic value of tumour and patient related factors in patients undergoing potentially curative resection of gastric cancer. Am J Surg. 2012;204(3):294-299. 
14. Cetin B, Berk V, Kaplan MA, et al. Is the pretreatment neutrophil to lymphocyte ratio an important prognostic parameter in patients with metastatic renal cell carcinoma? Clin Genitourin Cancer. 2013;11(2): $141-148$.

15. Teramukai S, Kitano T, Kishida Y, et al. Pretreatment neutrophil count as an independent prognostic factor in advanced non-small-cell lung cancer: an analysis of Japan Multinational Trial Organisation LC00-03. Eur J Cancer. 2009;45(11):1950-1958.

16. Cho H, Hur HW, Kim SW, et al. Pre-treatment neutrophil to lymphocyte ratio is elevated in epithelial ovarian cancer and predicts survival after treatment. Cancer Immunol Immunother. 2009;58(1):15-23.

17. Perez DR, Baser RE, Cavnar MJ, et al. Blood neutrophil-to-lymphocyte ratio is prognostic in gastrointestinal stromal tumor. Ann Surg Oncol. 2013;20(2):593-599.

18. Miettinen M, Lasota J. Gastrointestinal stromal tumors: pathology and prognosis at different sites. Semin Diagn Pathol. 2006;23(2):70-83.

19. Antonescu CR, Viale A, Sarran L, Tschernyavsky SJ, et al. Gene expression in gastrointestinal stromal tumors is distinguished by KIT genotype and anatomic site. Clin Cancer Res. 2004;10(10):3282-3290.

20. Curtis K, Youngquist ST. Part 21: categoric analysis: Pearson chi-square test. Air Med J. 2013;32(4):179-180.

21. Moghimi-Dehkordi B, Safaee A, Tabei SZ. A comparison between Cox proportional hazard models and logistic regression on prognostic factors in gastric cancer. East Afr J Public Health. 2009;6 Suppl(1):20-22.

22. Templeton AJ, McNamara MG1, Šeruga B, et al. Prognostic role of neutrophil-to-lymphocyte ratio in solid tumors: a systematic review and meta-analysis. J Natl Cancer Inst. 2014;106(6):dju124.

23. Özgehan G1, Kahramanca Ş, Kaya İO, et al. Neutrophil-lymphocyte ratio as a predictive factor for tumor staging in colorectal cancer. Turk J Med Sci. 2014;44(3):365-368.

24. Hanahan D, Weinberg RA. Hallmarks of cancer: the next generation. Cell. 2011;144(5):646-674.

25. Gooden MJ, de Bock GH, Leffers N, et al. The prognostic influence of tumour-infiltrating lymphocytes in cancer: a systematic review with meta-analysis. Br J Cancer. 2011;105(1):93-103.
26. Loi S, Sirtaine N, Piette F, et al. Prognostic and predictive value of tumor-infiltrating lymphocytes in a phase III randomized adjuvant breast cancer trial in node-positive breast cancer comparing the addition of docetaxel to doxorubicin with doxorubicin-based chemotherapy: BIG 02-98. J Clin Oncol. 2013;31(7):860-867.

27. Perez D, Hauswirth F, Jäger D, et al. Protein expression of cancer testis antigens predicts tumor recurrence and treatment response to imatinib in gastrointestinal stromal tumors. Int J Cancer. 2011;128(12): 2947-2952.

28. Petrie HT, Klassen LW, Kay HD. Inhibition of human cytotoxic T lymphocyte activity in vitro by autologous peripheral blood granulocytes. J Immunol. 1985;134(1):230234.

29. el-Hag A, Clark RA. Immunosuppression by activated human neutrophils. Dependence on the myeloperoxidase system. J Immunol. 1987; 139(7):2406-2413.

30. Di Carlo E, Forni G, Musiani P. Neutrophils in the antitumoral immune response. Chem Immunol Allergy. 2003;83:182-203.

31. McCourt M, Wang JH, Sookhai S, Redmond HP. Activated human neutrophils release hepatocyte growth factor/scatter factor. Eur J Surg Oncol. 2001;27(4):396-403.

32. Jabłońska E1, Kiluk M, Markiewicz W, et al. TNF-alpha, IL-6 and their soluble receptor serum levels and secretion by neutrophils in cancer patients. Arch Immunol Ther Exp (Warsz). 2001;49(1):63-69.

33. Schaider H, Oka M, Bogenrieder T, et al. Differential response of primary and metastatic melanomas to neutrophils attracted by IL- 8 . Int J Cancer. 2003;103(3):335-343.

34. Szkandera J, Absenger G, Liegl-Atzwanger B, et al. Elevated preoperative neutrophil/lymphocyte ratio is associated with poor prognosis in soft-tissue sarcoma patients. Br J Cancer. 2013;108(8):1677-1683.

35. Templeton AJ, Pezaro C, Omlin A, et al. Simple prognostic score for metastatic castration-resistant prostate cancer with incorporation of neutrophil-to-lymphocyte ratio. Cancer. 2014;120(21):3346-3352.
OncoTargets and Therapy

\section{Publish your work in this journal}

OncoTargets and Therapy is an international, peer-reviewed, open access journal focusing on the pathological basis of all cancers, potential targets for therapy and treatment protocols employed to improve the management of cancer patients. The journal also focuses on the impact of management programs and new therapeutic agents and protocols on

\section{Dovepress}

patient perspectives such as quality of life, adherence and satisfaction. The manuscript management system is completely online and includes a very quick and fair peer-review system, which is all easy to use. Visit http://www.dovepress.com/testimonials.php to read real quotes from published authors. 